\title{
Relationship between Mycobacterium Tuberculosis and Hookworm Infections among School Children in Mbita, Kenya
}

Manabu Inoue ${ }^{1 *}$, Sachiyo Nagi ${ }^{2}$, Evans Chadeka ${ }^{2}$, Faith Mutungi ${ }^{3}$, Mayuko Osada-Oka ${ }^{1,4}$, Kenji Ono ${ }^{5}$, Tetuya Oda ${ }^{5}$, Michinori Tanaka ${ }^{5}$, Yuriko Ozeki ${ }^{1,6}$, Kalenda Dan Justin Yombo ${ }^{2}$, Mayuko Okabe ${ }^{7}$, Mamiko Niki ${ }^{1}$, Yukio Hirayama ${ }^{1}$, Mitsuru Fukui ${ }^{8}$, Kazuo Kobayashi ${ }^{7}$, Makoto Matsumoto ${ }^{5}$, Masaaki Shimada ${ }^{9}$, Satoshi Kaneko ${ }^{9}$, Hisashi Ogura ${ }^{10}$, Yoshio Ichinose ${ }^{9}$, Sammy M Njenga ${ }^{3}$, Shinjiro Hamano ${ }^{2}$ and Sohkichi Matsumoto ${ }^{1,11^{*}}$ ${ }^{1}$ Departments of Bacteriology and Virology, Osaka City University Graduate School of Medicine, Japan

${ }^{2}$ Department of Parasitology, Institute of Tropical Medicine (NEKKEN) and the Global Center of Excellence, Nagasaki University, Japan

${ }^{3}$ Eastern and Southern Africa Centre of International Parasite Control (ESACIPAC), Kenya Medical Research Institute, Nairobi, Kenya

${ }^{4}$ Food Hygiene and Environmental Health Division of Applied Life Science, Graduate School of Life and Environmental Sciences, Kyoto Prefectural University, Japan

${ }^{5}$ Microbiological Research Institute, Otsuka Pharmaceutical, Kagasuno, Kawauchi-cho, Japan

${ }^{6}$ Department of Food and Nutrition, Sonoda Women's University, Japan

${ }^{7}$ Department of Immunology, National Institute of Infectious Diseases, Japan

${ }^{8}$ Department of Statistics, Osaka City University Graduate School of Medicine, Japan

${ }^{9}$ Nagasaki University Nairobi Research Station, NUITM-KEMRI Project, Nagasaki University, Japan

${ }^{10}$ Departments of Virology, Osaka City University Graduate School of Medicine, Japan

${ }^{11}$ Departments of Bacteriology, Niigata University School of Medicine, Japan

\begin{abstract}
Tuberculosis (TB) remains a serious threat for human health. The majority of TB cases arise from latent Mycobacterium tuberculosis infection (LTBI). Therefore, latent $M$. tuberculosis infection (LTBI) is a major reservoir of the pathogen, and every effort thus should be made to diagnose LTBI to ensure completion of the treatment of it. TB is endemic throughout most of the tropics, in which parasitic infections are prevalent as well. It was reported that Helminth infection, including hookworm, is a risk of active TB, but its effect on the establishment of LTBI is unknown. In this study, we conducted a cross-sectional survey of LTBI and parasitic infections among 240 children from schools situated along the shores of Lake Victoria in Mbita district, Kenya. Blood samples were analyzed for LTBI and enteric parasite infections. Among the 240 children examined, 75 (31.3 \%) were found to have LTBI. Of the 75 children with LTBI, 10 children (13.3\%) were found to be positive for hookworm eggs (odds ratio: 3.02 ; $95 \%$ confidence interval: 1.14-7.99). Our study suggests for the first time that hookworm infection is associated with not only active TB but also LTBI.
\end{abstract}

Keywords: Kenya; Hookworm; Neglected tropical diseases; Latent mycobacterium tuberculosis

\section{List of abbreviations}

LTBI: Latent Mycobacterium Tuberculosis Infection; TB: Tuberculosis; STH: Soil-Transmitted Helminthiasis; BCG: Bacille de Calmette et Guérin; MCV: Mean Cell Volume; MCH: Mean Cell Hemoglobin; MCHC: Mean Cell Hemoglobin Concentration; PBMCs: Preparation of peripheral Blood Mononuclear Cells; ESAT-6: Early Secreted Antigenic Target $6 \mathrm{kDa}$ protein; CFP10: 10kDa Culture Filtrate Protein; PPD: Purified Protein Derivative; ConA: Concavalin A; IFN- $\gamma$ : Interferon $\gamma$; Th2: T helper 2; Th1: T helper 1; IL-10: Interleukin-10

\section{Introduction}

Tuberculosis (TB) is a disease caused by Mycobacterium tuberculosis, an acid-fast bacillus and is a serious threat for human health. In 2010, 8.8 million people newly developed TB and 1.1 million people died from TB [1]. After the infection is established, the majority $(\sim 95 \%)$ of individuals does not develop the disease, but instead maintain longterm latent infection. Five to 10 percent of asymptomatically infected persons develop TB during their lifetime by endogenous reactivation. Therefore, Latent $M$. Tuberculosis Infection (LTBI) is a major reservoir of the pathogen, and every effort thus should be made to diagnose LTBI to begin treatment of it in order to reduce TB burden [2].

Parasitic infections are common in TB endemic areas and are likely to impact on the high burden of TB [3]. Among parasitic infections, neglected tropical diseases caused by parasites, such as filariasis, onchocerciasis, schistosomiasis, and Soil-Transmitted Helminthiasis (STH), are still serious problem for human health. A high prevalence of STH and schistosomiasis are closely related to poverty, poor environmental hygiene, and lifestyle [4]. It is estimated that $1.4,1$, and
1.3 billion people globally suffer from Ascaris lumbricoides, Trichuris trichiura, and hookworm, respectively. Seven hundred twenty million people infected with STH are estimated to have clinical symptoms, and approximately 135,000 people die from complications per year [5] Recent studies also suggest that chronic helminthic infection may reduce the efficacy of Bacille de Calmette et Guérin (BCG) vaccination, a live attenuated vaccine against TB $[5,6]$. Furthermore, regions with a high burden of helminthiasis are correlated with a high TB burden, implying that helminthic infection is a potential risk for TB development and vice versa [7]. However, the relationship between establishment of LTBI and each parasitic infection is unknown.

TB in children is public health problems with special significance, because children are more likely to progress life-threatening forms of the disease than adults. Besides, TB in children is a marker of recent transmission. However, information has been lacking about LTBI and

*Corresponding author: Manabu Inoue, Department of Bacteriology, Osaka City University Graduate School of Medicine, Japan, Tel: +81-6-6645-3745; Fax: +81-6-6645-3746; E-mail: m2074857@med.osaka-cu.ac.jp

Sohkichi Matsumoto, Department of Bacteriology, Osaka City University Graduate School of Medicine, Japan, Tel: +81-6-6645-3745; Fax: +81-6-66453746; E-mail: sohkichi@med.osaka-cu.ac.jp

Received September 02, 2013; Accepted October 18, 2013; Published October 21,2013

Citation: Inoue M, Nagi S, Chadeka E, Mutung F, Osada-Oka M (2013) Relationship between Mycobacterium Tuberculosis and Hookworm Infections among School Children in Mbita, Kenya. J Trop Dis 1: 120. doi: 10.4172/2329891X.1000120

Copyright: () 2013 Inoue M, et al. This is an open-access article distributed under the terms of the Creative Commons Attribution License, which permits unrestricted use, distribution, and reproduction in any medium, provided the original author and source are credited. 
its risk among children in $\mathrm{TB}$ endemic area, so far. Kenya has a large and rising TB burden [8] and it ranks 13th among the 22 high-TB burden countries in the world. The incidence of TB in Kenya was reported as 29,800 per 100,000 people in 2010 by the World Bank report released in 2011. A high prevalence of other infectious diseases, including parasitic infections, has been reported in Kenya as well [9-11]. Mbita is located on the lakeshore of Lake Victoria, Nyanza province, Western Kenya and is endemic area of several life threatening infectious diseases. In this study, we investigated the relationship between LTBI and parasitic infection among school-aged children in Mbita, Kenya.

\section{Materials and Methods}

\section{Ethical considerations}

Study procedures followed were in accordance with the ethical standards of the Helsinki Declaration and approved by the Scientific Steering and Ethical Review Committees of the Kenya Medical Research Institute (SSC No. 2084). All data collection activities were careful explained, and oral consent was obtained from the district authority and other opinion leaders (health personnel and education officials), the headmasters, and the representatives of the pupils' parents. Informed consent for the participation of children to the study was obtained from the parents/guardians. The consent form was written in English and translated into the local languages (Kiswahili and Luo) to obtain understanding for the study. Pupils whose parents/ guardians had given consent were provided with an explanation of the data collection activities and were allowed to not participate or drop out of the study if they chose. The parents or guardians of the children enrolled in the study did not incur any cost for the transport or processing of the samples.

\section{Study area, design and population}

This cross-sectional study was conducted in Mbita district located around Lake Victoria in Nyanza Province, western Kenya. The population in Mbita district is 55,929 [12]. The study target population comprised primary school children in standard four, aged 9 to 19 years. There are 64 primary schools in Mbita district. The total number of school children in standard four in the year 2011 was 1,747 (859 males and 888 females, District Education office; described in research proposal SSC1088). About aqueous supplies, $84 \%$ of the household use water of the lake. But particularly $53.2 \%$ of the students in Kombe who go to school in the city use the tap water. In the hygienic status, there were some differences between schools at the diffusion rate of the restroom.

Cluster sampling was used with primary schools as the clusters. Prior to randomization, information on the number and size of the schools in the study area was obtained from the district education authority and entered in a computerized database. Information of the longitude and latitude of each school was added to the database using mapping data, obtained using global positioning system hardware. Schools were ranked by geographical location to allow for equal distribution of the schools over the study area. Seven primary schools, including Kombe, Wanga, Nyawiya, Usungu, Ngodhe SDA, Wasaria, and Kamasengre, were randomly selected using $\mathrm{R}$ version 2. 14 . 0 software. Pupils in standard four in the selected schools, whose parents/ guardians agreed to the study, were enrolled in the study and assessed for BCG vaccination status and a history of ever being exposed to TB at home. Stool, urine and blood samples were collected from all registered individuals for diagnosis of the various infections.

\section{Exclusion criteria}

In this study, children were excluded if they did not complete an examination or the number of CD4-positive $\mathrm{T}$ cell counts was under 500 cells $/ \mathrm{ml}$. The reductions of CD4-positive $\mathrm{T}$ cell counts are more likely to indicate the infection of HIV.

\section{BCG vaccine inoculation career}

To know the status of previous vaccination with BCG, we checked the presence of BCG scars on the skins of the children.

\section{Detection of intestinal helminthic infection}

The children were appropriately instructed about the procedure for the collection of stool, after which they were given labeled specimen cups on the day before the test to collect stool in the next morning. The stool specimens for 3 consecutive days were transferred to a field lab and examined for hookworm eggs within an hour and intestinal helminthic infections by the Kato-Katz thick smear technique on the next day [13].

\section{Detection of Intestinal Protozoan Infection}

Intestinal protozoan infection was examined by the thin smear method using specimens collected as described above.

\section{Detection of Plasmodium falciparum, P. malariae, P. ovale, and $P$. vivax}

Plasmodium infection was identified by microscopic examination of a thin blood smear after staining with Giemsa. Four types of malaria parasites were distinguished by their morphological characteristics.

\section{Measurement of hematological indicators}

Blood samples were analyzed by using BM6050 (JEOL, Japan) to examine the amount of the hemoglobin, the number of red blood cells, and hematocrit. Mean Cell Volume (MCV), Cell Hemoglobin (MCH), and Cell Hemoglobin Concentration (MCHC) were calculated.

\section{Detection of LTBI}

PBMCs were purified from heparinized blood samples using BD Vacutainer cell preparation tubes (Becton, Dickinson and Company, Franklin Lakes, NJ USA 07417) according to the manufacturer's instructions. After blood drawing and inversion, cell preparation tubes were centrifuged at $1,800 \mathrm{rcf}$ for $20 \mathrm{~min}$ at room temperature. PBMCs were then collected, washed twice in Roswell Park Memorial Institute (RPMI) medium, and resuspended in $2.5 \mathrm{ml}$ of RPMI medium containing $10 \%$ FCS and $50 \mu \mathrm{M}$ 2-mercaptoethanol (RPMI complete medium).

M. tuberculosis infection was assessed by monitoring $\mathrm{T}$ cell responses to ESAT- 6 and CFP10, which are produced by $M$. tuberculosis complex but not by Mycobacterium avium-intracellular complex and BCG. We used overlapping synthetic peptides to detect $\mathrm{T}$ cell responses to ESAT6 and CFP10, because using these peptides can prevent the effect of nonspecific reactions due to contaminations of mitogens, such as LPS, and shorten the time to detect a reaction compared with use of recombinant proteins. The amino acid sequences of the synthetic peptides are as follows below.

ESAT-6 (1-20) (H-MTEQQWNFAGIEAAASAIQG-OH), ESAT-6 (11-30) (H-IEAAASAIQGNVTSIHSLLD-OH), ESAT6 (21-40) (H-NVTSIHSLLDEGKQSLTKLA-OH), ESAT6(31-50) (H-EGKQSLTKLAAAWGGSGSEA-OH), ESAT-6 (41-60) (H-AAWGGSGSEAYQGVQQKWDA-OH), ESAT6 (51-70) (H-YQGVQQKWDATATELNNALQ-OH), ESAT6 (61-80) (H-TATELNNALQNLARTISEAG-OH), ESAT-6 
$(71-90)$

(

ESAT-

CFP10 (H-MAEMKTDAATLAQEAGNFER-OH), (11-30) (H-LAQEAGNFERISGDLKTQID-OH), CFP10 (2140) (H-ISGDLKTQIDQVESTAGSLQ-OH), CFP10 (31-50) (H-GQVESTAGSLQGQWRGAAGTA-OH), CFP10 (41-

60) (H-GQWRGAAGTAAQAAVVRFQE-OH), CFP10 (5170) (H-AQAAVVRFQEAANKGKQELD-OH), CFP10 (6180) (H-AANKGKQELDEISTNIRQAG-OH), CFP10 (71-90) (H-EISTNIRQAGVQYSRADEEQ-OH), and CFP10 (81-100) (H-VQYSRADEEQQQALSSQMGF-OH) were synthesized. After purification with HPLC with over 95\% purity, peptides were dissolved with DMSO and mixed together to a final volume of $1 \mathrm{mg} / \mathrm{ml}$ for each peptide in each antigen.

PBMCs were cultured with PPD purchased from Japan BCG Laboratory, ConA was purchased from Sigma-Aldrich (Organic Materials and Fiber Sigma-Aldrich Corporation, St Louis, MO), and the synthetic peptides mixture was obtained according to standard procedures or without stimulants as negative controls. In brief, PBMCs derived from $250 \mu \mathrm{l}$ of blood were suspended in a half volume $(125 \mu \mathrm{l})$ of RPMI complete medium and seeded into the wells of 96-well roundbottomed tissue culture plates (Becton, Dickinson and Company, Franklin Lakes, NJ 07417). Another $125 \mu \mathrm{l}$ of RPMI complete medium, which contained each antigen peptide at a final concentration of $5 \mu \mathrm{g} /$ $\mathrm{ml}$ for ESAT6 and CFP10, and $10 \mu \mathrm{g} / \mathrm{ml}$ for ConA and PPD, was then added to the wells. The plates were incubated overnight at $37^{\circ} \mathrm{C}$ in a humidified atmosphere under $5 \% \mathrm{CO}_{2}$. The supernatants were collected and frozen at $-30^{\circ} \mathrm{C}$ until assay.

The amount of IFN- $\gamma$ in the supernatants was assessed by using the immune-chromatographic test, which was a house-made for research use in Otsuka (Paper preparation, Fukushima et al., Usefulness of the measurement of interferon-gamma in tuberculous pleural effusion and quantiferon samples by the immunochromatography test). Briefly, 50 $\mu \mathrm{l}$ of culture supernatant was added to the reaction tube and the test stick, in which anti-IFN- $\gamma$ antibody was embedded, was soaked in the supernatant. After 15 minutes, the test stick was transferred to the well of a plate containing $100 \mu \mathrm{l}$ of washing buffer. After the color of the background of the test stick disappeared, a positive line was visualized when over $125 \mathrm{pg} / \mathrm{ml}$ of IFN- $\gamma$ was contained in the supernatants.

\section{Data analysis}

Data were analyzed with Mann-Whiney test, Wilcoxon rank sum test, chi-square test and odds ratio by using IBM SPSS 20.0 software.

\section{Results}

In this study, we analyzed data from 240 school children. The demographic characteristics of the study population are shown in Table 1. One hundred sixty-seven students $(53.8 \%)$ were vaccinated with BCG by confirming BCG scars on their skins.

We evaluated LTBI in a comprehensive manner with conventional health assessment and in vitro-examination of $\mathrm{T}$ cell immune responses to ESAT6 and CFP10 that are unique protein antigens expressed by
M. tuberculosis, but not from BCG vaccine strains or the majority of non-tuberculosis mycobacteria. By examining culture supernatants of PBMCs derived from 240 students, who did not show fever or prolonged cough over 2 weeks by a health assessment, we detected more than $125 \mathrm{pg} / \mathrm{ml}$ of IFN- $\gamma$ in 191 children (79.6\%) when their PBMCs were stimulated with PPD and more than $125 \mathrm{pg} / \mathrm{ml}$ of IFN- $\gamma$ in 75 children (31.3\%) when stimulated with ESAT-6 and CFP10 (39 females and 36 males) (Figure 1). All responders to ESAT- 6 and CFP10 were included in the PPD responders. As predicted, BCG vaccination did not show a correlation with responses to ESAT6 and/or CFP10 ( $\mathrm{p}=0.11$ ). Based on these results, the children were assigned as individuals with LTBI when they did not show any symptom of TB and their PBMCs produced IFN- $\gamma$ upon being stimulated with ESAT6 and CFP10.

We simultaneously assessed infections with various parasites. There were no differences among schools at the prevalence of protozoal and parasitic infection except for S. mansoni. Table 2 summarizes the number of students found to be positive for hookworm, A. lumbricoides, T. trichiura, Schistosoma mansoni, Plasmodium falciparum, P. malariae, P. ovale, P. vivax, Entamoeba coli, E. histolytica/E. dispar/E. moshkovskii, Iodamoeba, Giardia intestinalis, Balantidium, Coccidia, and Blastocystis.

The association between LTBI and other parasitic infections was examined using the Mann-Whitney U-test, the Wilcoxon rank sum test, and the chi-square test. We found a significant association between LTBI and hookworm infection $(p=0.02)$. By contrast, we did not find any association between LTBI and other parasite infections. The $p$ values for comparison between LTBI and each parasite infection were $p=0.61$ for $A$. lumbricoides, $p=0.22$ for $T$. trichiura, $p=0.30$ for $S$. mansoni, $p=0.16$ for $P$. falciparum, $p=0.50$ for $P$. malariae, $p=1.00$ for $P$. ovale, $p=1.00$ for $P$. vivax, $p=0.71$ for $E$. coli, $p=0.86$ for $E$. histolytica $/ E$. dispar E. moshkovskii, $p=0.22$ for Iodamoeba, $p=0.28$ for $G$. intestinalis, $p=0.18$ for Balantidium, $p=0.21$ for Coccidia, and $p=0.68$ for Blastocystis (Table 2).

The numbers of individuals positive for hookworm and LTBI are shown in Table 2 together with their odds ratios with 95\% confidence intervals. The odds ratio of individuals infected with hookworm for LTBI was 3.019 times that in individuals without hookworm $(95 \%$ confidence interval: 1.14-7.99), indicating a possible association between hookworm infection with LTBI among school children.

\section{Discussion}

In this study, 75 out of 240 (31.3\%) students were defined as LTBI among the students of standard 4 in Mbita, Kenya. Ten students were infected with hookworm out of 75 individuals with LTBI, while there were eight students with hookworm out of 165 individuals without LTBI. The odds ratio of hookworm infection in the LTBI population was 3.019 times as high as that in the non-LTBI population.

Many parasitic infections, including helminthiasis, are called neglected tropical diseases, which are specially endemic in lowincome population in the developing countries but affect over one billion people [14]. The prevalence of hookworm infections, such as by Ancylostoma duodenale and Necator americanus, are estimated to

\begin{tabular}{|c|c|c|c|c|c|c|c|c|}
\hline Characteristics & $\mathbf{n}$ & $\begin{array}{l}\text { Kombe } \\
(n=26)\end{array}$ & $\begin{array}{l}\text { Wanga } \\
(n=25)\end{array}$ & $\begin{array}{l}\text { Nyawiya } \\
(n=40)\end{array}$ & $\begin{array}{l}\text { Usungu } \\
(n=39)\end{array}$ & $\begin{array}{c}\text { Ngodhe } \\
\text { SDA(n=25) }\end{array}$ & $\begin{array}{l}\text { Wasaria } \\
(n=57)\end{array}$ & $\begin{array}{l}\text { Kamasengre } \\
\quad(n=28)\end{array}$ \\
\hline Males & 105 & 14 & 12 & 19 & 16 & 9 & 24 & 11 \\
\hline Females & 135 & 12 & 13 & 21 & 23 & 16 & 33 & 17 \\
\hline Mean age & 240 & $11.8 \pm 1.27$ & $12.2 \pm 1.33$ & $11.8 \pm 1.77$ & $10.7 \pm 1.16$ & $11.4 \pm 1.60$ & $12.0 \pm 1.66$ & $12.3 \pm 2.44$ \\
\hline
\end{tabular}

Table 1: Number of school children in standard 4 examined in each school in Mbita, Kenya. 


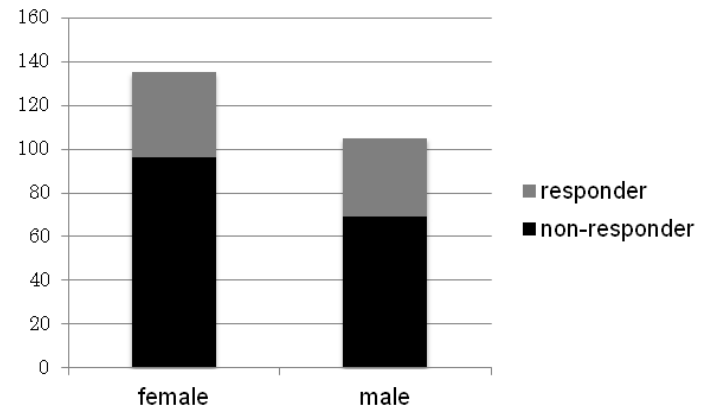

Figure 1: Number of asymptomatic ESAT6/CFP10 responders (LTBI population) and non-responders in male and female study subjects. The vertical axis indicates the number of students.

be frequent in developing countries, such as Asia, Africa, and Latin America, and approximately 740 million people are infected with these parasites in worldwide [14]. Infections with helminths, including hookworm, cause distinct immune responses. It is generally accepted that helminthic infection causes T helper 2 (Th2) immune responses. For example, Th2-dependent eosinophilic pneumonia is frequently observed after infection with a significant number of helminths, such as filaria, hookworm, or A. lumbricoides. However, some reports have shown that $\mathrm{T}$ helper 1 (Th1), rather than Th2 cytokines, are produced from PBMCs derived from hookworm infected-individuals in endemic areas [15-17]. In endemic areas, people are frequently exposed to pathogens that induce a Th1 response before or after they are infected with helminths, which may be the reason why Th2 immune responses appear dampened, even in those co-infected with helminthes. Infection with protozoa or bacteria that can stimulate a Th1 response results in suppression of the Th2 response induced by helminthic infection in mouse models $[15,18,19]$.

On the other hand, it was reported that co-infection of both helminths and $M$. tuberculosis results in the suppression of IFN- $\gamma$ production [20]. The Th2 response caused by hookworm infections may suppress a protective Th1 response against $M$. tuberculosis infection, and therefore, hookworm infection may allow the survival of $M$. tuberculosis and vice versa. Because we detected LTBI by measuring IFN- $\gamma$, an indicator of Th1 response, we cannot deny the possibility that LTBI-negative 8 children who infected with hookworm are falsenegative for LTBI. Taking into account this possibility, our data indicates all the more significant association between LTBI and hookworm.

In our study, only hookworm infection was correlated with LTBI, in spite of the fact that other helminths, such as $A$. lumbricoides or T. trichiura, can evoke robust Th2 responses, as well as in the hookworm infection. In addition, we did not find the significant increase of the level of peripheral eosinophils, which are index of the Th2 immune response compared with those in uninfected children in this study. It is likely that the shift of an immune response from Th1 to Th2 caused by helminthic infections is not a sole reason to explain higher ratio of LTBI in hookworm-infected students.

Immunosuppressive $\mathrm{T}$ cells, such as regulatory $\mathrm{T}$ cells (Treg), suppress cellular immune responses through direct contact with immune effector cells and by the production of regulatory cytokines, including TGF- $\beta$ and IL-10 [21,22]. It has been reported that hookworm infection causes induction of $\mathrm{CD} 4^{+} \mathrm{CD} 25^{+} \mathrm{FOXP} 3^{+} \mathrm{IL}-10^{+}$ regulatory T cells [23] and repeated infection with hookworm stimulates production of high levels of IL-10 [24], which inhibits host protective immunity against M. tuberculosis. Accordingly, there is a possibility that hookworm-induced IL-10 impacts on the sensibility to M. tuberculosis infection.

It is generally known that iron-deficiency anemia is caused by heavy infection with adult hookworms in the intestinal tract. It is estimated that 36 million out of 1,300 million people infected with hookworm have iron-deficiency anemia, and 65,000 people annually die from hookworm-induced anemia. Iron-deficiency anemia is a typical symptom observed in individuals heavily infected with hookworm, but rarely seen in those with other helminths. Iron status also affects TB disease; for example, iron overload is reported to be a risk for TB progression [25]. In this study, the MCV and MCHC of the students, which are indicators of iron-deficiency anemia, were lower than the standard values generally, but there was no difference between hookworm-infected and non-infected children (MCV: $p=0.35$; MCHC: $p=0.47$ ), and there was no difference in iron status between responders to ESAT6 and/or CFP10 and non-responders $(p=0.16)$. Accordingly we did not observe obvious effects of anemia caused by helminth infection on the establishment of LTBI in this study.

The current study found that hookworm infection was associated with LTBI. As discussed above, the exact reason of this finding is unclear. In mice model, recent report showed that Mycobacterium-specific both Th1 and Th17 cells by hookworm infection enhances establishment of LTBI [26], supporting our human study. We consider that next studies should examine whether this association can be observed in other area and adults as well. Addictively, the studies should be conducted to examine whether hookworm infection causes expansion of the LTBI population and/or directly contributes to TB progression in addition to establishment of infection because there are several reports indicating that helminthic infection is a risk factor for developing TB [27-29]. Our study suggests the necessity of the deep studies to understand the relationship between $M$. tuberculosis and hookworm infection on the prevalence and progression of disease in high-burden countries, including tropical and subtropical areas.

\section{Acknowledgements}

This work was supported by grants from the Ministry of Education, Culture Sports, Science and Technology; the Ministry of Health, Labour and Welfare (Research on Emerging and Re-emerging Infectious Diseases, Health Sciences Research Grants); The Japan Health Sciences Foundation; The United StatesJapan Cooperative Medical Science Program against Tuberculosis and Leprosy; and Collaborative Research Foundation of Otsuka. We are grateful to Ms. Sara Matsumoto, Chihiro Inoue, and Inori Omura for assistance with the experiments. This paper is published with the permission of Director KEMRI.

\section{References}

1. [No authors listed] (2010) WHO global tuberculosis control report 2010 Summary. Cent Eur J Public Health 18: 237

2. Druszczyńska M, Kowalewicz-Kulbat M, Fol M, Włodarczyk M, Rudnicka W (2012) Latent M. tuberculosis infection--pathogenesis, diagnosis, treatment and prevention strategies. Pol J Microbiol 61: 3-10.

3. Boraschi D, Abebe Alemayehu M, Aseffa A, Chiodi F, Chisi J (2008) Immunity against HIVIAIDS, malaria, and tuberculosis during co-infections with neglected infectious diseases: recommendations for the European Union research priorities. PLoS Negl Trop Dis 2: e255.

4. Crompton DW (1999) How much human helminthiasis is there in the world? J Parasitol 85: 397-403.

5. Elias D, Britton S, Aseffa A, Engers H, Akuffo H (2008) Poor immunogenicity of BCG in helminth infected population is associated with increased in vitro TGFbeta production. Vaccine 26: 3897-3902

6. Barreto ML, Rodrigues LC, Silva RC, Assis AM, Reis MG, et al. (2000) Lower 
Citation: Inoue M, Nagi S, Chadeka E, Mutung F, Osada-Oka M (2013) Relationship between Mycobacterium Tuberculosis and Hookworm Infections among School Children in Mbita, Kenya. J Trop Dis 1: 120. doi:10.4172/2329-891X.1000120

hookworm incidence, prevalence, and intensity of infection in children with a Bacillus Calmette-Guérin vaccination scar. J Infect Dis 182: 1800-1803.

7. Hotez PJ, Molyneux DH, Fenwick A, Ottesen E, Ehrlich Sachs S, et al. (2006) Incorporating a rapid-impact package for neglected tropical diseases with programs for HIVIAIDS, tuberculosis, and malaria. PLoS Med 3: e102.

8. van't Hoog AH, Laserson KF, Githui WA, Meme HK, Agaya JA, et al. (2011) High prevalence of pulmonary tuberculosis and inadequate case finding in rural western Kenya. Am J Respir Crit Care Med 183: 1245-1253.

9. Pullan RL, Gething PW, Smith JL, Mwandawiro CS, Sturrock HJ, et al. (2011) Spatial modelling of soil-transmitted helminth infections in Kenya: a disease control planning tool. PLoS Negl Trop Dis 5: e958.

10. Njenga SM, Mwandawiro CS, Muniu E, Mwanje MT, Haji FM (2011) Adult population as potential reservoir of NTD infections in rural villages of Kwale district, Coastal Kenya: implications for preventive chemotherapy interventions policy. Parasit Vectors 4: 175

11. Clements AC, Deville MA, Ndayishimiye O, Brooker S, Fenwick A (2010) Spatial co-distribution of neglected tropical diseases in the east African great lakes region: revisiting the justification for integrated control. Trop Med Int Health 15: 198-207.

12. Kaneko S, K'opiyo J, Kiche I, Wanyua S, Goto K, et al. (2012) Health and Demographic Surveillance System in the Western and coastal areas of Kenya: an infrastructure for epidemiologic studies in Africa. J Epidemiol 22: 276-285.

13. Katz N, Chaves A, Pellegrino J (1972) A simple device for quantitative stool thick-smear technique in Schistosomiasis mansoni. Rev Inst Med Trop Sao Paulo 14: 397-400

14. Hotez PJ, Brooker S, Bethony JM, Bottazzi ME, Loukas A, et al. (2004) Hookworm infection. N Engl J Med 351: 799-807.

15. Hoeve MA, Mylonas KJ, Fairlie-Clarke KJ, Mahajan SM, Allen JE, et al. (2009) Plasmodium chabaudi limits early Nippostrongylus brasiliensis-induced pulmonary immune activation and Th2 polarization in co-infected mice. BMC Immunol 10: 60 .

16. Quinnell RJ, Pritchard DI, Raiko A, Brown AP, Shaw MA (2004) Immune responses in human necatoriasis: association between interleukin-5 responses and resistance to reinfection. J Infect Dis 190: 430-438.

17. Wright V, Bickle $Q$ (2005) Immune responses following experimental human hookworm infection. Clin Exp Immunol 142: 398-403.
18. Liesenfeld O, Dunay IR, Erb KJ (2004) Infection with Toxoplasma gondii reduces established and developing Th2 responses induced by Nippostrongylus brasiliensis infection. Infect Immun 72: 3812-3822.

19. Buendia A, Fallon PG, Del Rio L, Ortega N, Caro MR (2002) Previous infection with the nematode Nippostrongylus brasiliensis alters the immune specific response against Chlamydophila abortus infection. Microb Pathog 33: 7-15.

20. Resende Co T, Hirsch CS, Toossi Z, Dietze R, Ribeiro-Rodrigues R (2007) Intestinal helminth co-infection has a negative impact on both antiMycobacterium tuberculosis immunity and clinical response to tuberculosis therapy. Clin Exp Immunol 147: 45-52.

21. Bueno LL, Morais CG, Araujo FF, Gomes JA, Correa-Oliveira R (2010) Plasmodium vivax: induction of $C D 4+C D 25+F o x P 3+$ regulatory $T$ cells during infection are directly associated with level of circulating parasites. PLoS One 5: e9623.

22. Belkaid $Y$ (2007) Regulatory T cells and infection: a dangerous necessity. Nat Rev Immunol 7: 875-888.

23. Ricci ND, Fiuza JA, Bueno LL, Cancado GG, Gazzinelli-Guimaraes PH (2011) Induction of CD4(+)CD25(+)FOXP3(+) regulatory T cells during human hookworm infection modulates antigen-mediated lymphocyte proliferation. PLoS Negl Trop Dis 5: e1383.

24. Geiger SM, Massara CL, Bethony J, Soboslay PT, Corrêa-Oliveira R (2004) Cellular responses and cytokine production in post-treatment hookworm patients from an endemic area in Brazil. Clin Exp Immunol 136: 334-340.

25. Boelaert JR, Vandecasteele SJ, Appelberg R, Gordeuk VR (2007) The effect of the host's iron status on tuberculosis. J Infect Dis 195: 1745-1753.

26. George PJ, Anuradha R, Kumaran PP, Chandrasekaran V, Nutman TB, et al. (2013) Modulation of mycobacterial-specific Th1 and Th17 cells in latent tuberculosis by coincident hookworm infection. J Immunol 190: 5161-5168.

27. Gangaidzo IT, Moyo VM, Mvundura E, Aggrey G, Murphree NL, et al. (2001) Association of pulmonary tuberculosis with increased dietary iron. $\mathrm{J}$ Infect Dis 184: 936-939.

28. Elias D, Mengistu G, Akuffo H, Britton S (2006) Are intestinal helminths risk factors for developing active tuberculosis? Trop Med Int Health 11: 551-558.

29. Brown M, Miiro G, Nkurunziza P, Watera C, Quigley MA, et al. (2006) Schistosoma mansoni, nematode infections, and progression to active tuberculosis among HIV1-infected Ugandans. Am J Trop Med Hyg 74: 819-825. 